\title{
ZONE-BASED ENERGY EFFICIENT ROUTING PROTOCOLS FOR WIRELESS SENSOR NETWORKS
}

\author{
RAJAN SHARMA* BALWINDER SOHI ${ }^{\dagger}$ AND NITTIN MITTAL
}

\begin{abstract}
This paper proposes a novel zone or grid-based network deployment framework for energy efficient selection and reselection process of Zone-Head $(\mathrm{ZH})$ in the WSNs. The proposed zone head reselection process ensures energy efficiency, load balancing, and stability which further prolongs the network lifetime. Instead of carrying out periodic reselection of Zone-Head (ZH) that leads to extra energy consumption and network overhead, the protocol dynamically initiates the process of reselection based on residual energy level of $\mathrm{ZH}$. In the proposed approach the process is segregated into four phases; deployment phase, the zone formation phase, zone head selection phase, data transmission phase and reselection phase. We implemented the proposed algorithm in MATLAB and its result outcomes reveal that the proposed method outperforms the competitive algorithms for parameters such as load balancing, total energy consumption and network lifetime.
\end{abstract}

Key words: DRESEP, SEECP, WSN, Residual energy, Network lifetime

AMS subject classifications. $68 \mathrm{M} 10,68 \mathrm{M} 12$

1. Introduction. In Wireless Sensor Networks (WSNs), routing protocol plays key role in increasing network energy efficiency and is a source of inspiration for those researchers who attempts to save the energy of wireless sensor node and to enhance the lifetime of the network in parallel [1]. The operation which consumes most of the energy in WSNs is the activity of data packet routing. The characteristics of the WSNs are unique in contrast to traditional networks. These unique characteristics are often taken into account for addressing the issues and challenges related to network coverage, node distribution, node administration, network deployment, energy efficiency, security [2-4] and so forth. In recent years, WSNs have been widely investigated [3- 5]. WSN typically consists of large number of low cost unattended multifunctioning sensing nodes that are typically deployed in large quantities and in a high density manner with limited energy resource [1]. These sensing nodes are linked by wireless medium using radio, infrared, or optical frequency band. These networks have various applications like flood and fire detection in remote areas, traffic surveillance, air traffic control, and so forth. Sensors jointly gather ambient condition information such as temperature, pressure, and humidity from their surrounding environment and forward it towards static data sink. In many scenarios, as nodes are deployed in remote and dangerous area, replacement of their batteries becomes impossible. So they must work without replacing their batteries for many years [6]. Thus power management has become one of the fundamental issues of WSNs. The factors which causes energy consumption in WSNs and deteriorates the network lifetime are collision, overhead, overhearing, idle listening, complexity and traffic fluctuations. These factors deplete the energy resources of WSNs. In WSNs, single-hop routing consumes more energy and leads to unbalancing the energy distribution to the nodes which are far from base station (BS). On the other hand, limited radio range of the node and other environmental factors (obstacles, noise, interference, etc.) make single-hop communication infeasible [7]. In WSNs nodes are often randomly distributed across a given geographical area. In such situation some regions in the network get densely populated whereas others receive less number of nodes. Cluster and grid-based algorithms [8-9] are employed to cope up with this problem. Cluster based schemes minimize energy consumption and simplify network management by treating related nodes in groups. Cluster-based approaches increase scalability, robustness, provide load balancing and data aggregation [1, 6, 9]. These algorithms are utilized for clustering in efficient manner where the entire terrain is segregated into virtual grids, and are widely used because of its simplicity, scalability, and uniformity in energy consumption across the network [10]. In literature, different energy efficient cluster-based and grid-based algorithms have been proposed such as LEACH [11], TEEN [12], CBDAS and GBDD [13] but still load balancing and energy efficiency are open issues because of the randomized nature of WSN. The iterative process of cluster formation and $\mathrm{CH}$ reselection requires transmitting continuous control messages which results in extensive energy consumption of the nodes

*Research Scholar, IKG Punjab Technical University, Kapurthala, Jalandhar.(rajansharma.ece@gmail.com).
${ }^{\dagger}$ CGC Group of Colleges.(balsohi18@gmail.com)

$\ddagger$ Chandigarh University.(mittal.nitin84@gmail.com) 
and leads to poor performance of the network. This paper focuses on a grid or zone-based technique that can ensure load balancing and intelligent selection and reselection of zone head (ZH) to maximize network lifetime. Our main contribution is to develop a robust network model, which has been developed to deal with variability in deployment area and node density. The network is divided into equal squared size grids and the number of nodes in each zone is determined by their coordinates. Dual-hop communication strategy between ZH and BS is used to attain load balancing. After the topology construction, ZH selected is the one having the maximum energy level and distance value. The role of $\mathrm{ZH}$ is rotated based on some threshold to increase network stability and overall operational lifetime. This paper is segregated as follows. Section 2 describes about related work. The Section 3 and 4 are focused on the network model and proposed ZEERP protocol respectively. Simulation results of ZEERP are discussed in Section 5. Stability-aware model of ZEERP is given in Section 6 and its performance is presented in Section 7. In section 8, the proposed work is concluded with future scope.

2. Related work. The main operational sustainability concern in WSN is its energy resource constraint. In recent years, numerous routing protocols have been proposed for WSNs based on the network organization and the routing protocol operations [6]. Some of these focused on minimizing the communication distance to reduce the energy consumption and a handful of them focused on fair energy distribution to avoid the hot spot problems. In existing literature, many researchers have proposed different clustering techniques that are briefly discussed below. Heinzelman et al. presented a low energy efficient adaptive clustering hierarchy (LEACH) algorithm in which sensor nodes arrange themselves into clusters for data aggregation [11]. In this protocol, data aggregation is performed periodically by cluster heads $(\mathrm{CHs})$ to reduce the redundancy in information to be communicated to BS. In setup phase, $\mathrm{CHs}$ are nominated on the basis of two parameters; percentage of $\mathrm{CH}$ selection and number of times a particular node has been appointed as $\mathrm{CH}$. To make a decision, a random number (between 0 and 1 ) is chosen by the node. To become a $\mathrm{CH}$ for the present round, the number picked by node should be less than threshold value. During steady state phase, the data transmission takes place between non $\mathrm{CHs}$ to $\mathrm{CH}$, and then data is transmitted finally to BS. LEACH-centralized (LEACH-C) [14] uses BS as a centralized point to create clusters in an optimal manner. The overhead of collecting clustering information at BS to form the clusters is a drawback of LEACH-C. Manjeshwar et al. presented a reactive clustering algorithm named threshold-sensitive energy efficient sensor network (TEEN). It senses the medium continuously and transfers the data less frequently [12]. In TEEN, the network consists of homogeneous nodes and is divided into two level CHs. First level and second level CHs are distinguished in such a way that the $\mathrm{CHs}$ which are far from BS are termed as first level $\mathrm{CHs}$ and the closer ones are called second level CHs. It is efficient for the time-critical data sensing applications. In this, a CH sends its members the values of hard and soft threshold [12]. Once hard threshold value is achieved, nodes forward the data to CH. The subsequent data is transmitted only when the environment changes by a minimum of soft threshold value. The main drawback of TEEN is that sensor nodes can never transfer data to BS if the threshold values are not achieved. To get better performance, stable election protocol (SEP) (Smaragdakis, Matta, \& Bestavros, 2004) is proposed to maintain the hierarchical routing in WSNs where two types of nodes have their own election probability. Kumar et al. presented a clustering protocol named EEHC in the heterogeneous model [16]. The network is divided into three categories according to the initial energy of nodes; normal, advanced and super nodes. Apparently, normal nodes have the least energy, and super nodes have the highest level of energy. EEHC is based on SEP, and the three types of nodes in EEHC have their own election probability to be CHs within a fixed time to keep stable. Energy efficient and scalable sensor network can be achieved through clustering and multi-hop transmission. It has attracted much attention of the researchers. In [17], Kumar et al. improved EEHC further and proposed a multi-hop clustering protocol called MCR. In MCR, the multi-hop path is built to reduce the energy consumption. Mittal et al. proposed a clustering algorithm using dual-hop communication between $\mathrm{CH}$ and BS, suitable for event driven applications called DRESEP [18] and its stability-aware algorithm named SEECP [19] to improve its stability period. Researchers combined the cluster scheme with the biologically inspired routing scheme, and they proposed the evolutionary algorithms (EAs). The EAs are used to handle the cluster-based problem to minimize energy consumption and improve network lifetime with heterogeneity [20-23]. These routing schemes which are inspired by EAs demonstrated their advantages in prolonging the lifetime of hierarchical WSNs. The techniques discussed here are used to minimize the energy consumption of network. Besides all these amenities, clustering leads to hotspot problem in which certain number of sensors 
expire early because of their excessive usage. This results in network partitioning and polarization of nodes. In Grid-based clustering, the whole network area is divided into virtual grids. In GBDD [13], the network is divided into grids (also called cells) initiated by the BS. The first node interested in communicating data is set as the crossing point for the grid and its coordinates become the reference point for the grid creation. In this approach, it is often difficult to achieve preferred number of grids required by the network scenario. In a similar approach, the whole network is partitioned into grids based on the node location where midpoints are computed using the membership degree [24-25]. In another approach, the network is divided into two levels of square shaped grids; low level and high level [26]. Low level is for in-cluster data gathering whereas high level is used for inter-cluster data transmission. In unequal clustering mechanism, whole network is segregated into variable size clusters in which $\mathrm{CH}$ reserves more energy for inter-cluster communication in order to avoid hotspot problem [27]. Authors have used multi-hop energy aware routing scheme to balance and minimize the energy load of the $\mathrm{CH}$ for inter-cluster communication. Variable size clustering algorithms can result in balanced energy consumption maximizing network lifetime. However, extra advertisements for cluster head selection may lead to extra computation and energy overhead. There are various protocols, techniques and algorithms designs which saves the energy of WSNs. Distance-based Residual Energy-efficient Stable Election Protocol (DRESEP) [18] and its stability-aware version named Stable Energy Efficient Clustering Protocol (SEECP) [19] are energy efficient solutions in WSNs. In [28], proposed a Hierarchical Energy Efficient MAC protocol (HEEMAC) which combines the supremacy of LEACH and CSMA approach. This protocol utilizes the concept of CSMA based data transmission along with neighbor acknowledgement (N-Ack). Results proves that proposed protocol delivers high throughput, energy saving and prolonging lifetime of network. In [29], authors proposed an ANN based framework for evaluating optimal cost routing using BB-BC optimization approach in the WSNs. The integrated link cost is a function of average delay from end to end, residual energy of node and throughput of the sensor network. Authors focused on the literature review of variety of MAC protocols for WSNs based upon different parameters such as energy efficiency, throughput delay, and packet loss [30]. In the literature, we observed that the main aim is to keep the wireless sensor nodes in energy saving mode to the extreme extent and to minimize the parameters which are source of energy wastage such as re-transmission, congestion, overhear, idle channel sensing and overhead due to control packets so that lifespan of network can be prolonged. R. Sharma et al. investigates the impact of DVFS and DMS schemes on energy consumption and lifetime of sensor node [31]. In [32-37], aithors focused on performance, energy efficiency and secured solutions in WSNs. Looking at the above discussion, we can summarize that the services provided by different clustering techniques still have several shortcomings that need to be addressed, for instance, network management overhead, hotspot problem, and broadcasting issues. The above discussion also shows that grid-based system is a better option but the dynamic nature of sensor networks makes it difficult to predict the size of grids and number of nodes. In the proposed technique, the problems of hotspot, non-uniform distribution of nodes (load balancing), and computation overhead have been addressed. Furthermore, the proposed technique is not only energy efficient but also performs better on load balancing when compared with state-of-the-art techniques.

3. Energy dissipation radio model. Power consumption is one of the key factors in designing WSN protocols as sensor nodes are highly energy limited. Sensors consume energy for data processing, wireless communication and sensing data. The network energy is consumed on both sides of the communication (transmission and reception) according to a wireless energy consumption model as shown in Figure 3.1 [11, 18, 22]. The model consists of two parts reflecting transmission and reception as shown in equation 1 and 2 respectively. SN needs to consume energy E TX to run the transmitter circuit and E amp to activate the transmitter amplifier, whereas a receiver consumes E RX power for running the receiver circuit. Energy consumption in wireless communication also depends on message length (1) [18, 23]. The transmission cost for a l-bit message having communication distance $\mathrm{d}$, is calculated as

$$
E_{T X}=\left\{\begin{array}{cc}
l E_{\text {elec }}+l \varepsilon_{\text {friss_amp }} d^{2}, & \text { if } d<d_{0} \\
l E_{\text {elec }}+l \varepsilon_{\text {two_ray_amp }} d^{4}, & \text { if } d \geq d_{0}
\end{array}\right.
$$

where $d_{0}$ is crossover distance and is given by:

$$
d_{0}=\sqrt{\varepsilon_{\text {friss_amp }} / \varepsilon_{\text {two_ray_amp }}}
$$




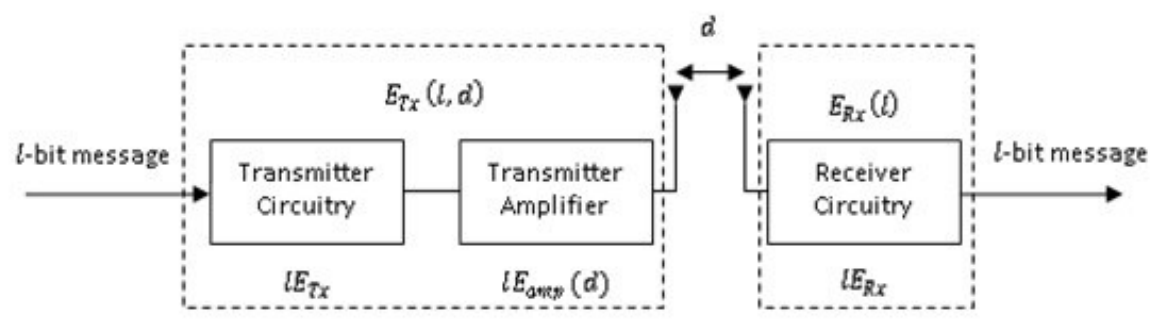

FiG. 3.1. Energy radio model diagram.

The term $E_{\text {elec }}$ signifies the per-bit energy expended for transmission. The parameters $\varepsilon_{\text {friss_amp }}$ and $\varepsilon_{t w o \_r a y \_a m p}$ represent energy expended to run radio amplifier in free space and two ray ground propagation models, respectively. The reception cost for the $l$-bit data message is given by:

$$
E_{R X}=l E_{\text {elec }}
$$

where $E_{\text {elec }}$ is the per-bit energy consumption for reception.

4. ZEERP. To address the problem of load balancing and energy consumption mentioned in the previous section, we proposegrid or zone-based network framework named Zone-based energy efficient routing protocol (ZEERP). It is a reactive, load balancing and energy efficient protocol for WSNs. The proposed approach evenlydistributes the load across the network, improves networkmanagement, and extends network lifetime. Figure 4.1 presents the proposed framework. The process is segregated into the following main phases.

(i) Deployment Phase: The total number of nodes (where $=1,2,3, \ldots$. , ) are randomly deployed in a square targeted area $(\mathrm{A}=\mathrm{FH} \times \mathrm{FW})$, where and are the field height and width, respectively. We assume some default node parameters, for instance, coordinates, node ID, and energy level. Once the topology is built and nodes are deployed, they share this configuration information with the BS. This information is later used by BS for carrying out the grid formation procedure more efficiently.

(ii) Zone Formation Phase: In this phase, the data collected from different sensors is used to form zones and construct topology as presented in Algorithm 1. The grid denotes a single zone recognized by unique zone ID. Once the zone formation phase is completed, BS governs the number of nodes per zone by computing the initial and finish point of each zone as mentioned in Algorithm 1. Figure ??shows zone formation where nodes are randomly deployed across grids. In this figure, $\mathrm{C} 0$ to $\mathrm{Cm}-1$ are the columns and $\mathrm{R} 0$ to Rm-1 represent rows. (Zxs, Zys) and (Zxe, Zye) represent the start and end of each zone. Zone height $\left(Z_{H}\right)$ and zone width $\left(Z_{W}\right)$ of each grid are calculated as

$$
\begin{aligned}
& Z_{H}=F_{W} / M \\
& Z_{W}=F_{W} / M
\end{aligned}
$$

where $F_{H}$ and $F_{W}$ represent the height and width of field.

(iii) Zone Head Selection Phase: Zone Head (ZH) selection is very crucial for any energy efficient protocol. ZH is responsible for processing or making any decision upon the received data. ZH selection is an important process; therefore, it is required to define criteria before selection of the ZH. The performance of a zone depends on the ZH; therefore, it is significant to elect the best node as the $\mathrm{ZH}$ among available nodes. In the proposed technique two parameters: residual energy level $R_{E}$ and average distance value $A_{D}$ are aggregated to come up with $\mathrm{ZH}$ election value $\left(Z H_{E V}\right)$ of a single node i as follows:

$$
Z H_{E V}(i)=0.5 * R_{E}(i)+0.5 *\left(1 / A_{D}(i)\right)
$$

The energy level of the node $\mathrm{i}$ is represented by $R_{E}$; initially it will be the same for all nodes. Higher value of $R_{E}$ increases the chance of the candidate node for becoming the ZH. $A_{D}$ is the average distance 


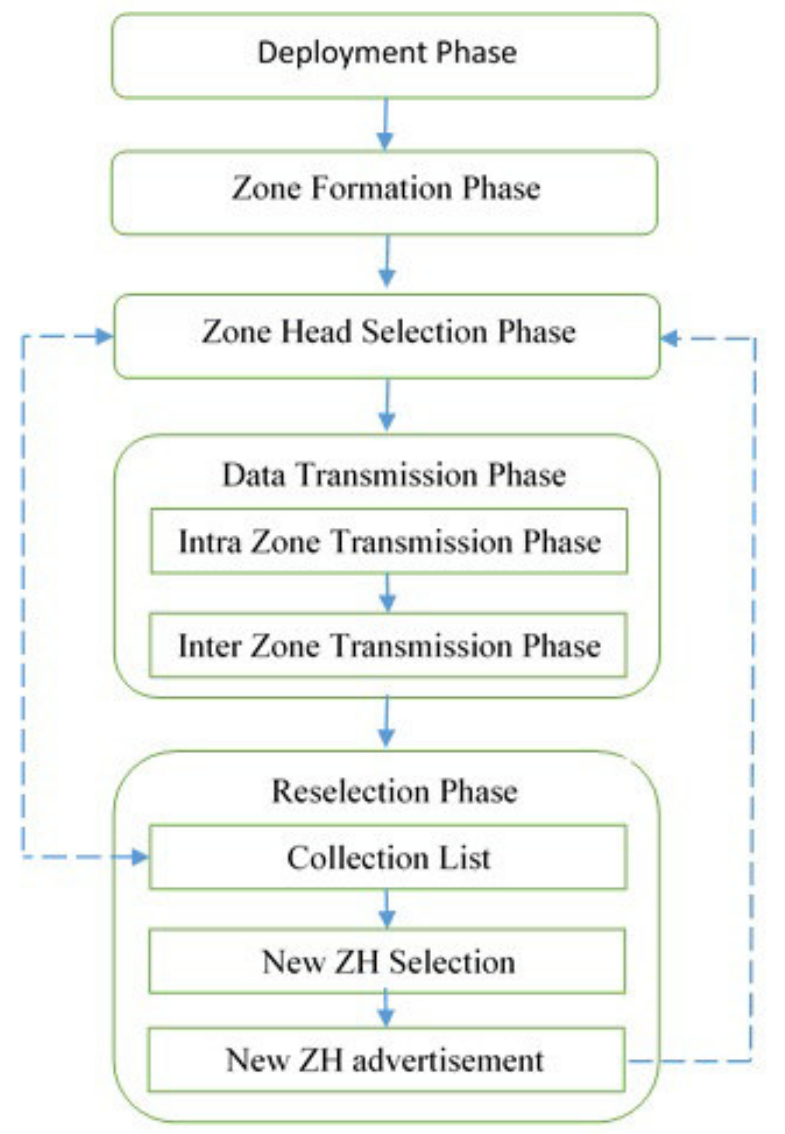

FIG. 4.1. Framework of the proposed technique.

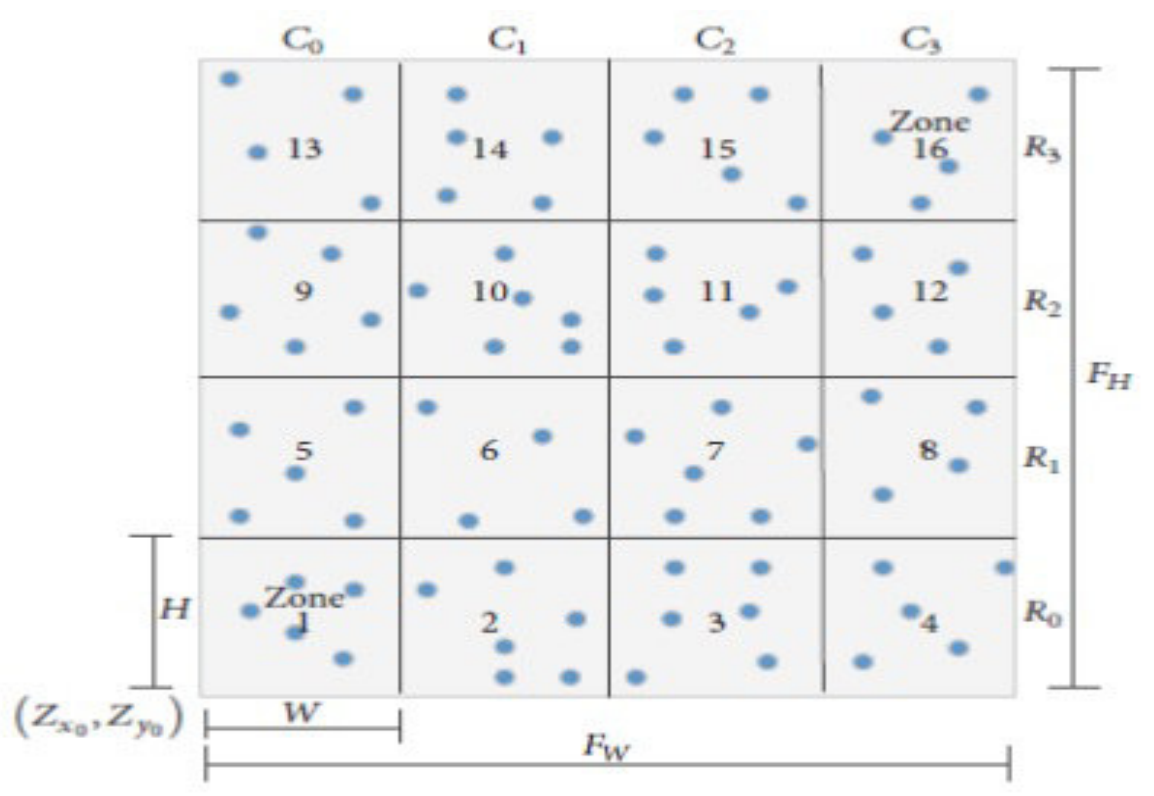

FIG. 4.2. Zone formation. 


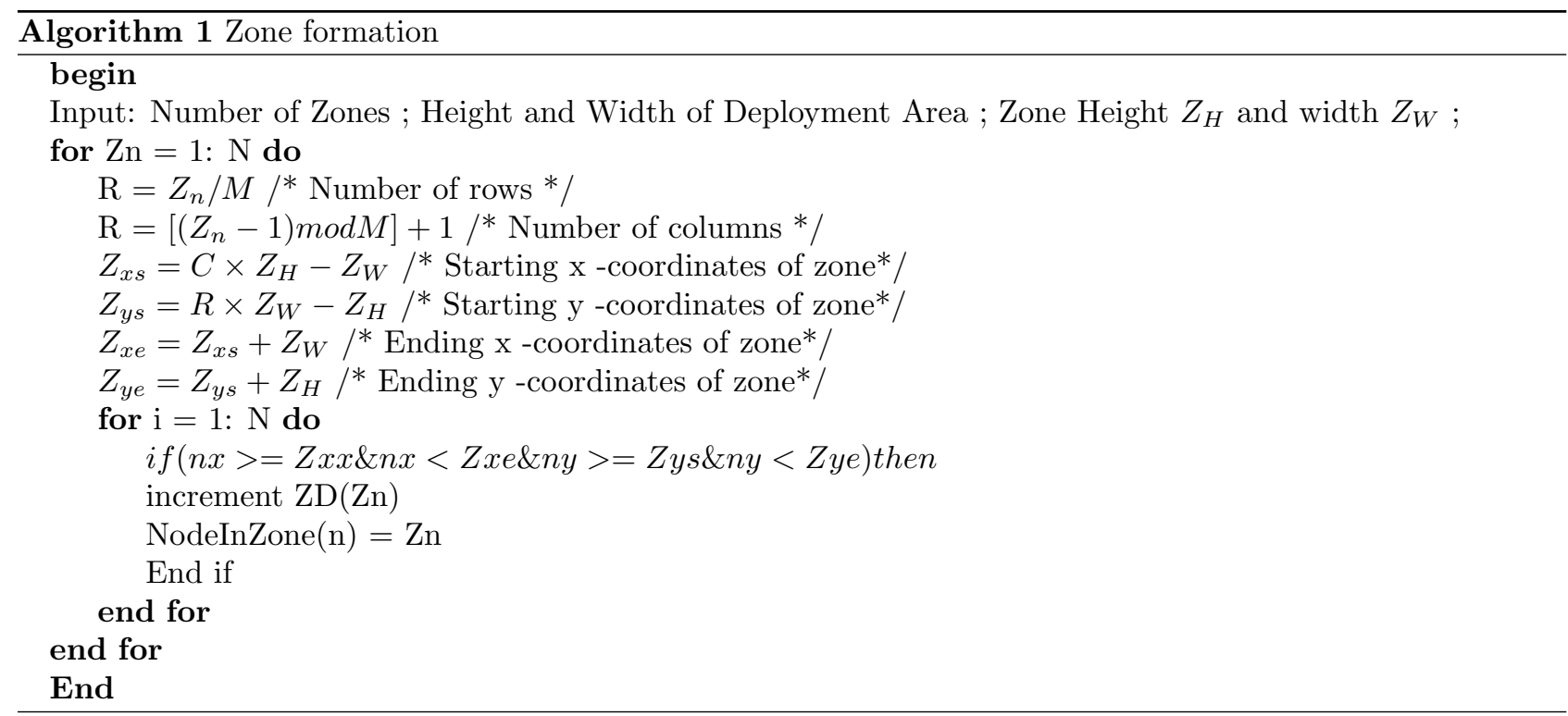

value of each individual node in that specific zone as given in (7). $A_{D}$ is the distance of a node from all other nodes within the zone and from center of the zone as shown in (8) and (9), respectively. Minimum value of $A_{D}$, calculated by $\mathrm{BS}$, will increase nodes chance to be a $\mathrm{ZH}$. BS will get the $A_{D}$ of all nodes within the network, which will be calculated once

$$
A_{D}(i)=\operatorname{cent}(i, c)+\frac{1}{n-1} \sum_{j=1}^{n} d(i, j), j \neq i
$$

where $d(i, j)$ is distance of a node from other nodes in its zone. In order to know how far a node is from other nodes which are in direct transmission with it, consider

$$
d(i, j)=\sqrt{(i x-j x)^{2}+(i y-j y)^{2}}
$$

wherecent $(i, c)$ is the center of zone. In order to know the position of the node in its zone, consider

$$
\operatorname{cent}(i, c)=\sqrt{(i x-c x)^{2}+(i y-c y)^{2}}
$$

Once the $\mathrm{ZH}$ criteria are set and zones are formed, $\mathrm{ZH}$ is selected for each individual zone according to (6). Base station will have the collection list that will have $Z H_{E V}$ of all nodes against each zone in the network. Node with maximum $Z H_{E V}$ will be selected as $\mathrm{ZH}$ for that specific zone. The nominated ZHs announce their status to the network using advertisement message that contains its ID. Each node replies the join request to $\mathrm{ZH}$. Each $\mathrm{ZH}$ builds a TDMA plan for its zone members to permit their communication. It specifies time slots during which zone members need to be in active state only when they are authorized to transmit the data.

The main advantage of having a collection list is to avoid any broadcast and communication of any maintenance messages during reselection process of $\mathrm{ZH}$. The reselection process is decentralized where the base station is not involved. This approach significantly reduces the number of messages exchanged in the reselection process of $\mathrm{ZH}$ in a zone eventually reducing energy consumption and thus maximizing network lifetime. The lifetime of a $\mathrm{ZH}$ for one complete iteration is determined by a threshold valuediscussed in reselection phase.

(iv) Data Transmission Phase: Once nodes join ZH, it senses the environment continuously. Each non-ZH node switch ON their radio and transmit their sensed information to their respective head only when 


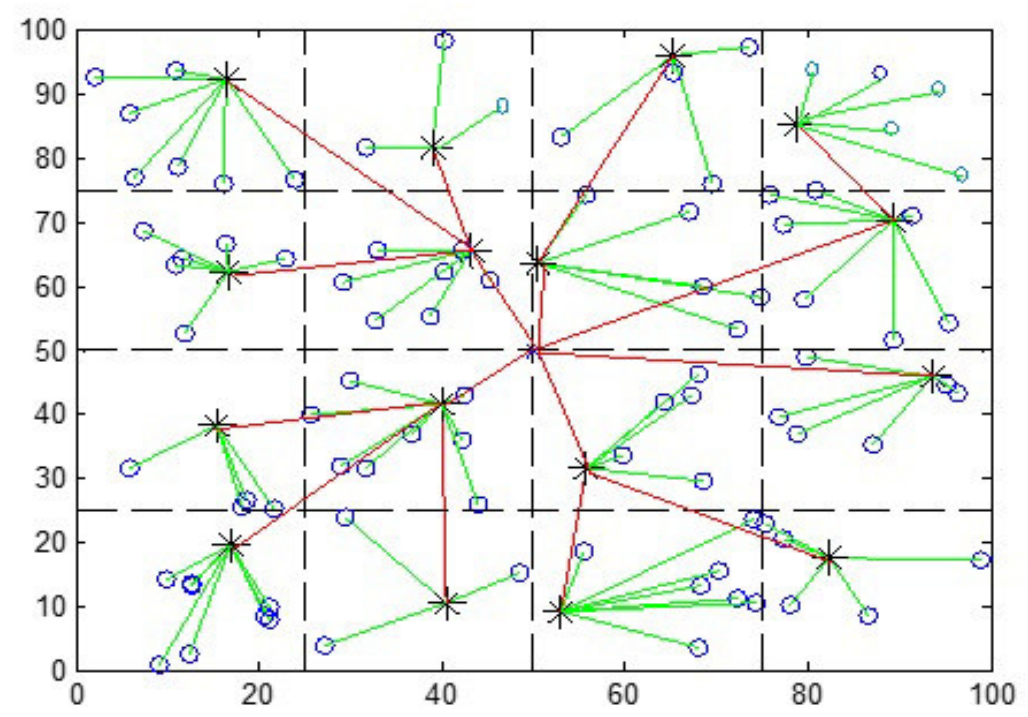

FIG. 5.1. Zone formation and data transmission for ZEERP simulation network.

an event is activated (i.e. current sensed value surpasses hard threshold value $H(T)$ ), Once $H(T)$ value is attained, the subsequent transmission is feasible if variation in sensed value surpasses the soft threshold value S(T) (Mittal \& Singh, 2015)-(Mittal, Singh, \& Sohi, 2017). The nodes send their sensed data to the $\mathrm{ZH}$ as per their assigned TDMA schedule of transmission. This enables nodes to keep their radio off until its transmission time occurs. The sleep periods save node energy. In wakeup periods, ZHs will aggregate and compress the received data. ZHs transmit their aggregated information to BS using direct or dual-hop basis as a function of distance between them (Mittal \& Singh, 2015), (Mittal, Singh, \& Sohi, 2017).

(v) Reselection Phase: In this phase, the attention is to minimize the energy consumption in reselection process of ZH. Instead of carrying out periodic reselection of $\mathrm{ZH}$ that leads to extra energy depletion and network overhead, the protocol dynamically initiates the process of reselection based on the election value $Z H_{E V}$ of the $\mathrm{ZH}$. In a given iteration, if the $Z H_{E V}$ value is less than or equal to average energy of the respective zone, the corresponding $\mathrm{ZH}$ will change. The number of iterations is independent of the zone and the reselection is carried out per zone when required. The number of iterations can be different for each zone to minimize the traffic generated in the network and also not to disturb the overall network. In order to select the new $\mathrm{ZH}$ in next round, the value of average zone energy is therefore periodically monitored by ZH. For this purpose every $\mathrm{ZH}$ maintains the collection list that contains $Z H_{E V}$ and residual energy of nodes.

5. Performance analysis of ZEERP. The performance of proposed algorithm is evaluated by carrying out extensive simulations. All simulations were carried out using MATLAB R2015a. The performance of ZEERP is compared with several state-of-the-art energy efficient cluster-based protocols including LEACH,TEEN and DRESEP.

This research work is carried out to investigate the effects of proposed algorithm in homogeneous setup. 100 nodes are randomly deployed in a $100 \mathrm{~m} \times 100 \mathrm{~m}$ area with an initial energy of $E_{0}=1 J$ as presented in Figure 5.1 Non-ZH nodes are represented by 'o' and ZHs are represented by '*'. The parameters setting for proposed protocol is given in Table 5.1 .

The simulation results reveal that in ZEERP, total energy consumption during zone formation,ZH selection, reselection of $\mathrm{ZH}$, and transmission has been reduced. To analyze the network lifespan against each method, various simulations were run for initial energy of $1 \mathrm{~J}$ for 100 nodes and packet size of 4000 bits. In Figure 5.2, it is 
TABLE 5.1

Simulation parameters used for ZEERP.

\begin{tabular}{|l|l|}
\hline Parameter & Value \\
\hline Number of nodes & 100 \\
\hline Network size & $100 \mathrm{~m} \times 100 \mathrm{~m}$ \\
\hline Location of BS & $(50,50)$ \\
\hline Initial energy, $E_{0}$ & $1 \mathrm{~J}$ \\
\hline Grid Size & $4 \times 4$ \\
\hline Radio electronics energy, $E_{T x}=E_{R x}$ & $50 \mathrm{~nJ} / \mathrm{bit}$ \\
\hline Energy for data-aggregation, $E_{D A}$ & $5 \mathrm{~nJ} / \mathrm{bit}$ \\
\hline Radio amplifier energy, $\varepsilon_{\text {friss_amp }}$ & $100 \mathrm{pJ} / \mathrm{bit} / \mathrm{m}^{2}$ \\
\hline Radio amplifier energy, $\varepsilon_{\text {two_ray_amp }}$ & $0.0013 \mathrm{pJ} / \mathrm{bit} / \mathrm{m}^{4}$ \\
\hline Temperature range on the field & $0^{\circ} \mathrm{F}-200^{\circ} \mathrm{F}$ \\
\hline Hard threshold & $50^{\circ} \mathrm{F}$ \\
\hline Soft threshold & $2^{\circ} \mathrm{F}$ \\
\hline
\end{tabular}

Number of Alive Nodes per Round

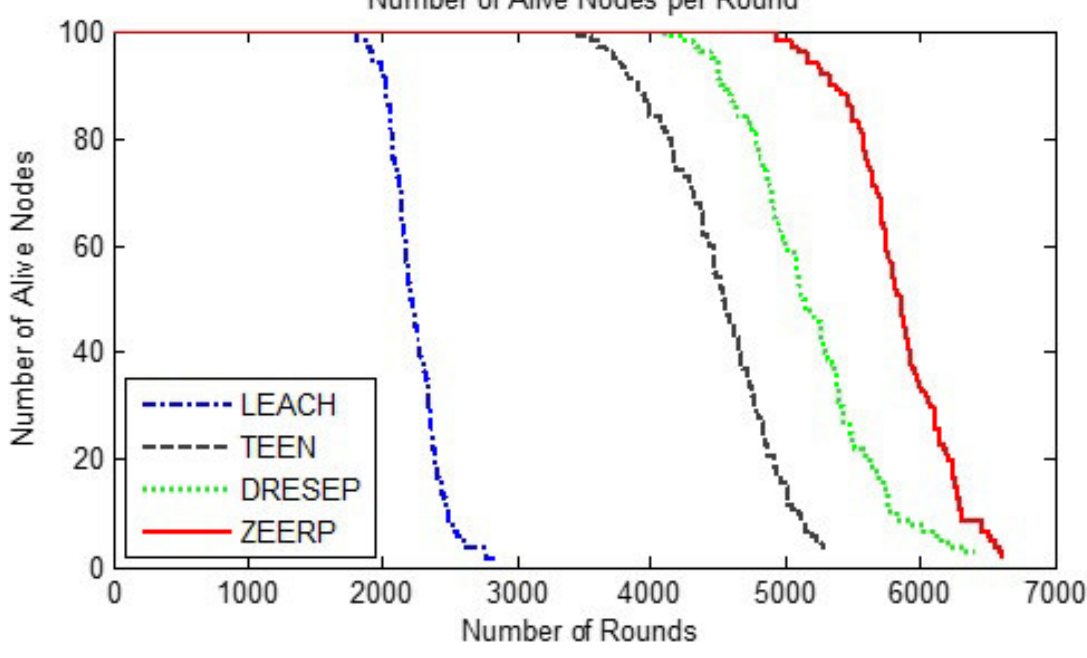

6 .

FIG. 5.2. Network lifetime comparison for LEACH, TEEN, DRESEP and ZEERP.

clear that ZEERP surpasses the competitive algorithms in terms of energy efficiency and prolong lifetime. This is because the control messages in ZEERP are reduced in ZH selection and reselection process. This increases the number of rounds and maximizes network lifetime. Table 5.2 shows the round history of dead nodes and average network lifetime in terms of number of rounds it takes until first node dies (FND), half of nodes die (HND) and last node dies (LND) for homogeneous setup. The lifetime comparison of ZEERP protocol with respect to FND, HND and LND is shown in Figure 5.3.

Figure 5.4 shows total energy consumed against the number of rounds. The graph shows that energy consumed by the proposed protocol is less than others with increase in number of rounds. This is due to the even distribution of nodes across the network resulting in steady energy consumption. In comparison with LEACH, TEEN and DRESEP, the proposed protocol maximizes the lifetime approximately by $225.14 \%, 12.12 \%$ and $2.12 \%$ respectively.

To evaluate the impact of grid size on the performance of ZEERP, whole network is partitioned into grid sizes of $4 \times 4,6 \times 6$, and $8 \times 8$ grids by keeping the same parameters such as number of nodes (100) with initial energy $E_{0}=1 J$. In all three approaches, the proposed technique with grid size $8 \times 8$ has achieved maximum number of rounds thereby improving network lifetime as illustrated in Figure 5.5. This technique is 
TABLE 5.2

Round history of dead nodes for simulated protocols

\begin{tabular}{|l|l|l|l|l|}
\hline \% dead Nodes & LEACH & TEEN & DRESEP & ZEERP \\
\hline 1 (FND) & 1805.2 & 3518.3 & 4101.6 & $\mathbf{4 9 2 3 . 5}$ \\
\hline 10 & 2022.8 & 4048.3 & 4504.2 & $\mathbf{5 3 2 5 . 8}$ \\
\hline 20 & 2069.3 & 4228.9 & 4769.9 & $\mathbf{5 5 6 7 . 5}$ \\
\hline 30 & 2141 & 4379.2 & 4881.4 & $\mathbf{5 6 7 0 . 1}$ \\
\hline 40 & 2169.4 & 4460.7 & 4983.5 & $\mathbf{5 7 3 2 . 3}$ \\
\hline $50(\mathrm{HND})$ & 2215.2 & 4569.6 & 5126.7 & $\mathbf{5 8 5 1 . 4}$ \\
\hline 60 & 2280.1 & 4731.5 & 5294.2 & $\mathbf{5 9 1 4 . 2}$ \\
\hline 70 & 2346.3 & 4820.1 & 5395.4 & $\mathbf{6 0 6 6}$ \\
\hline 80 & 2394.8 & 4972.3 & 5621.1 & $\mathbf{6 1 9 8 . 3}$ \\
\hline 90 & 2485.6 & 5154.7 & 5770.7 & $\mathbf{6 2 9 1 . 2}$ \\
\hline $100(\mathrm{LND})$ & 2763.5 & 5388.2 & 6402.2 & $\mathbf{6 5 7 6 . 8}$ \\
\hline
\end{tabular}

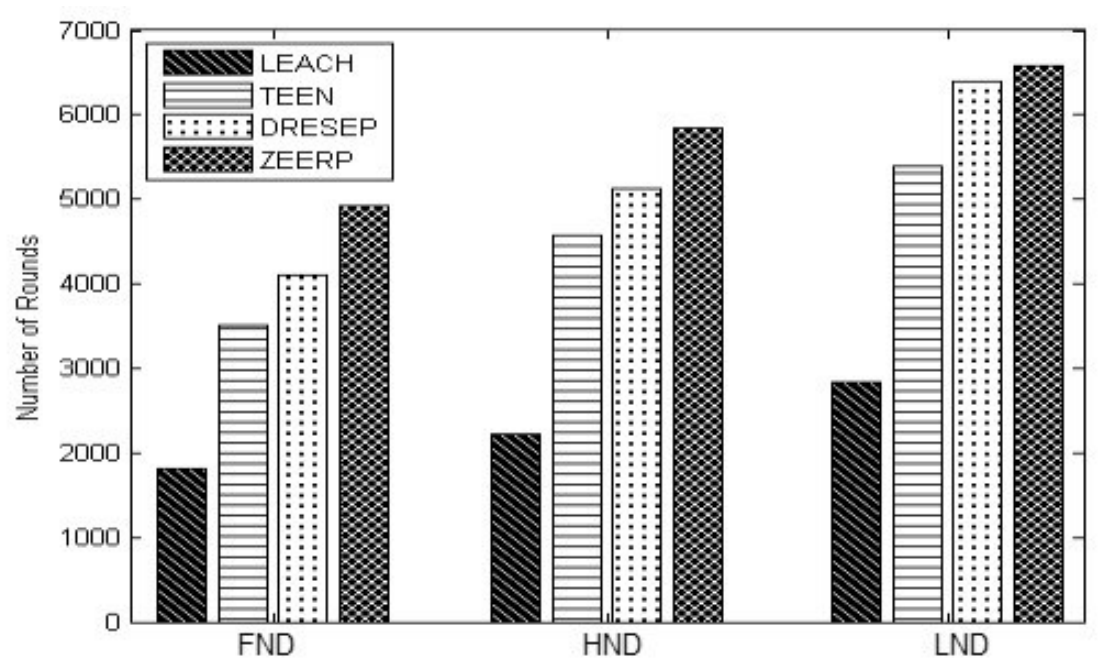

FIG. 5.3. Lifetime comparison of simulated protocols for homogeneous set-up.

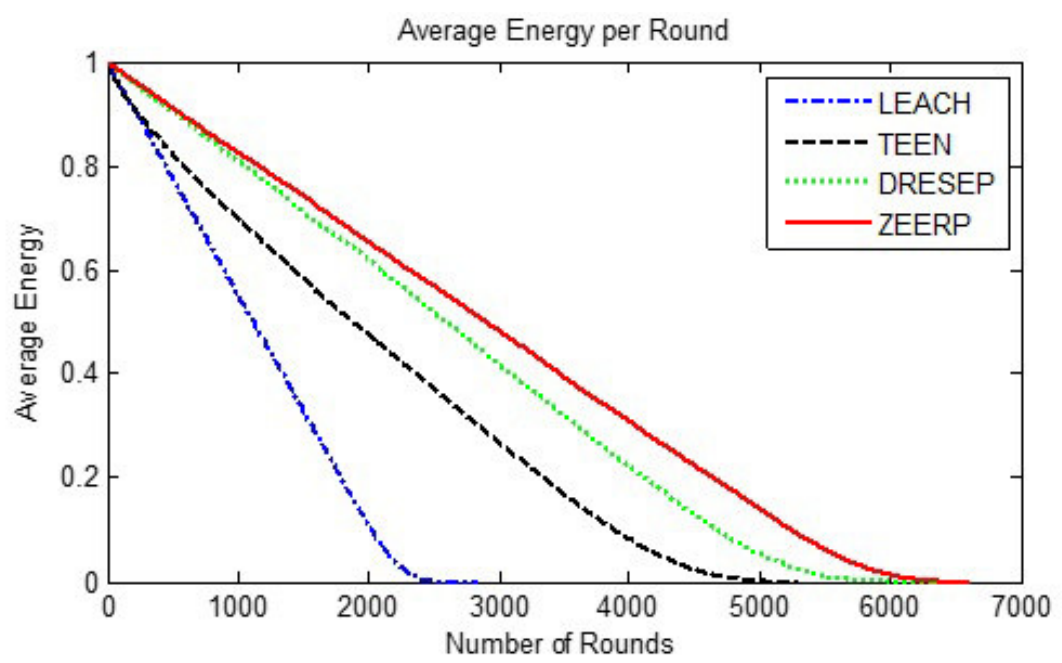

FIG. 5.4. Average energy per round for simulated protocols. 


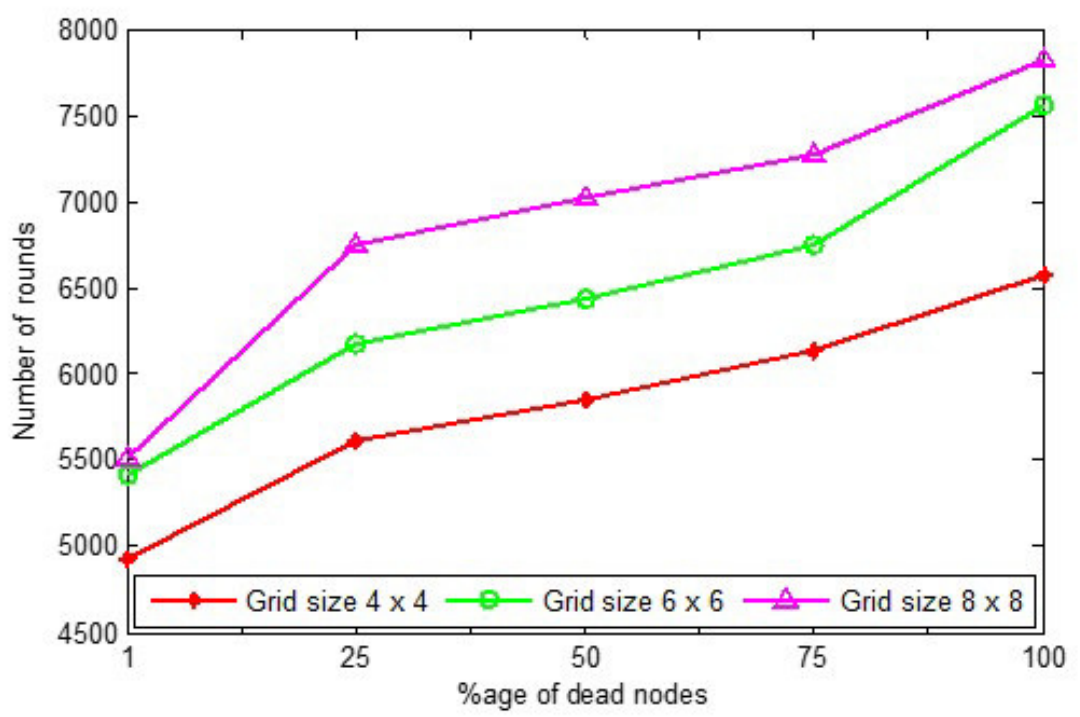

FIG. 5.5. Effect of grid size on the performance of ZEERP

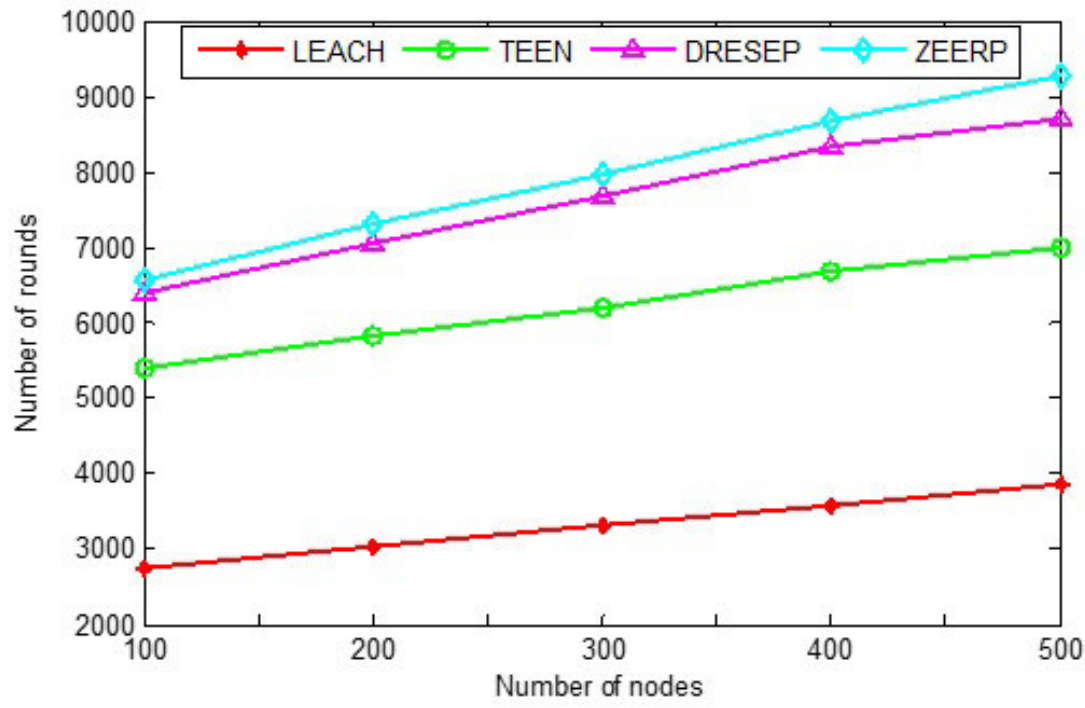

FIG. 5.6. Effect of node density on the performance of ZEERP

approximately 1.1 and 1.2 times better for grid sizes $4 \times 4$ and $6 \times 6$ respectively.

To evaluate the effect of node density in each protocol, the number of nodes is varied from 100 to 500 with initial energy $1 \mathrm{~J}$ as shown in Figure 5.6. The number of rounds increases by increasing the node density. ZEERP has more number of rounds than other approaches for varying node density. By increasing the node density, the resulting network has better lifetime as the responsibilities of each ZH is distributed.

The initial energy of sensor nodes is set to $0.25 \mathrm{~J}, 0.5 \mathrm{~J}$, and $1.0 \mathrm{~J}$ for evaluating LEACH, TEEN, DRESEP and ZEERP to determine the number of rounds when $1 \%, 25 \%, 50 \%, 75 \%$, and $100 \%$ nodes of the network die. Figures 5.7, 5.8 and 5.9 show that the proposed algorithm has larger number of rounds, this is because the control messages are reduced in ZH selection and reselection process. This increases the number of rounds and maximizes network lifetime. 


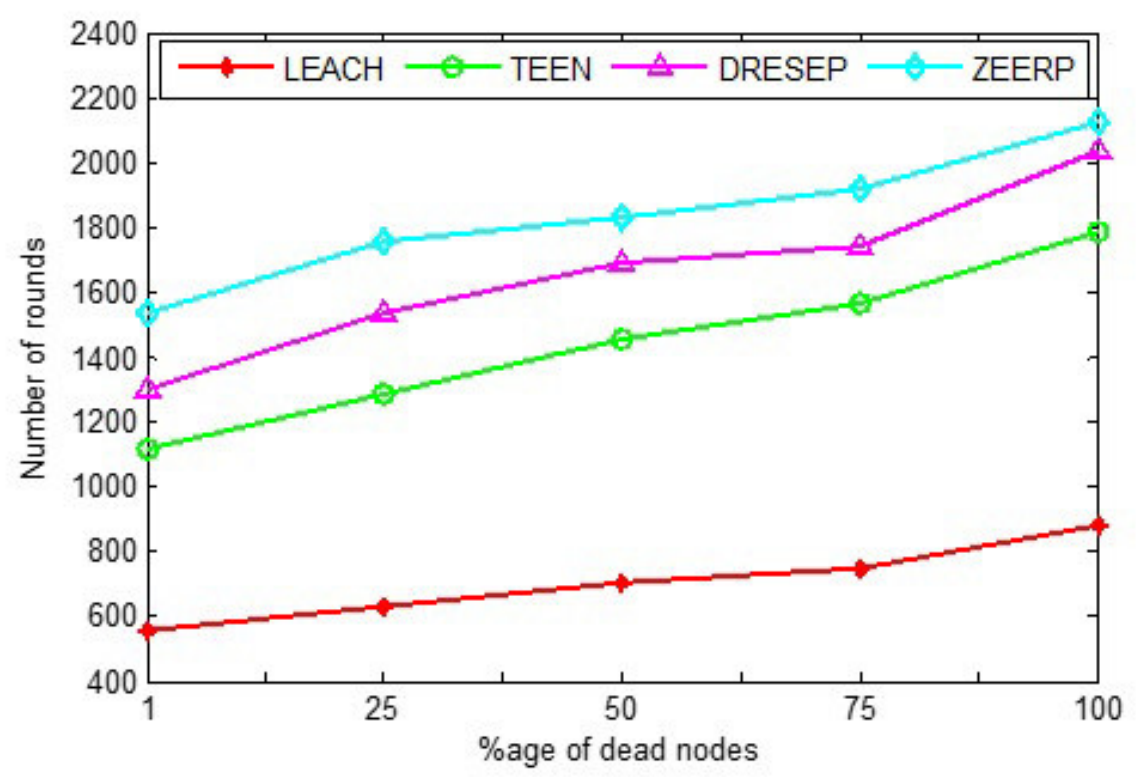

FIG. 5.7. Number of rounds when 1with initial energy $E 0=0.25 \mathrm{~J}$.

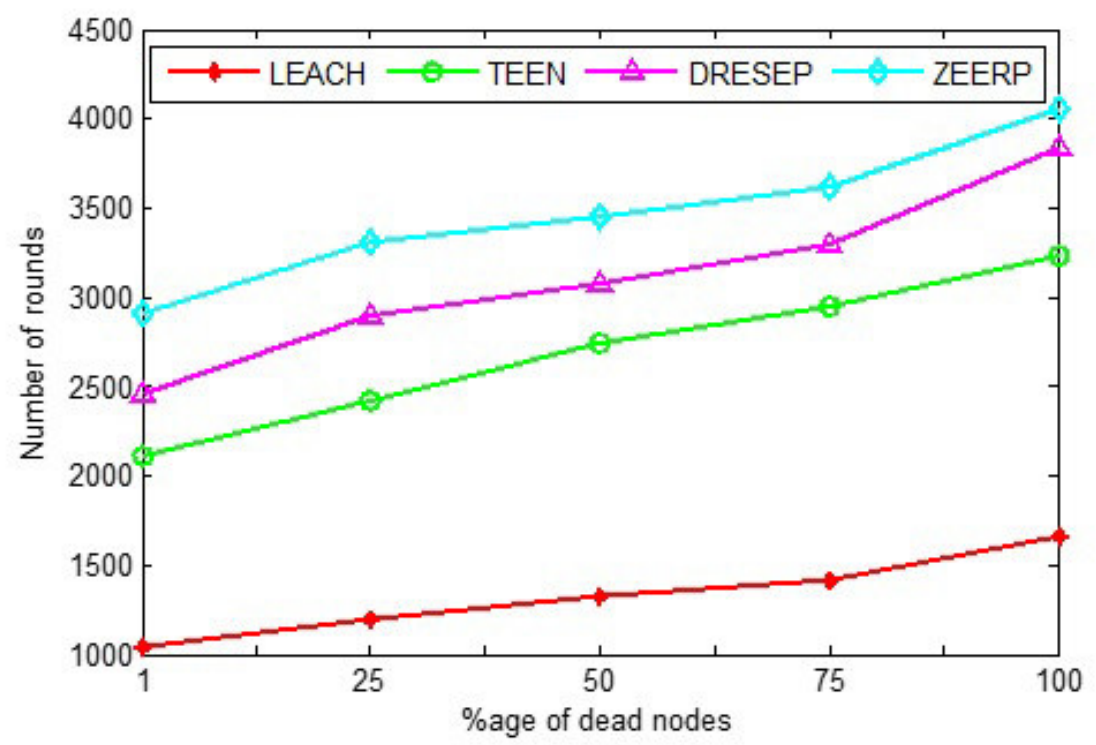

FIG. 5.8. Number of rounds when 1\%, 25\%, 50\%, 75\%, and $100 \%$ nodes die in the network with initial energy E $0=0.5 \mathrm{~J}$.

6. Stability-aware ZEERP. By a careful analysis of ZEERP, one can see that while this protocol succeeded in prolonging the WSN lifetime but is failed to ensure a longer stability (i.e., reliable) period until FND and reduced instability period (difference between LND and FND). For applications such as agricultural monitoring and smart environments, the protocol aims at maximizing the total lifetime of the application while minimizing the energy consumed by participating SNs. For crucial applications such as environmental monitoring, factory automation and security, the protocol aims at maximizing the stability period of the application 


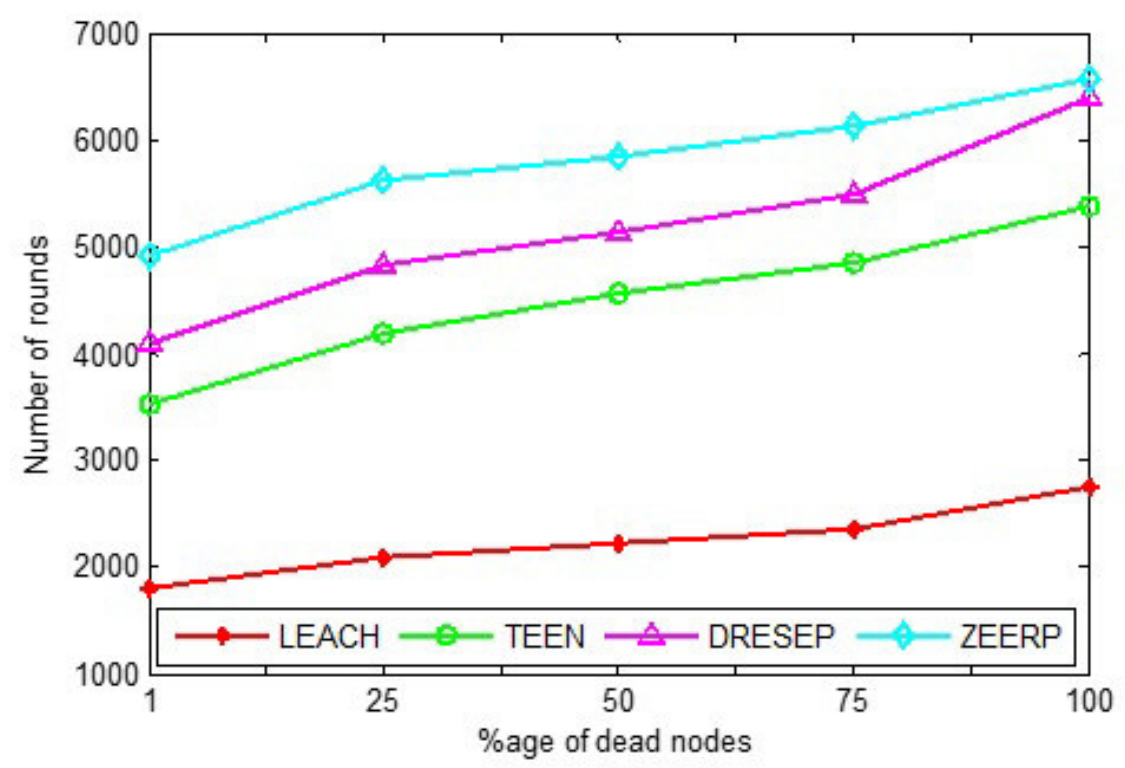

FIG. 5.9. Number of rounds when 1\%, 25\%, 50\%, 75\%, and $100 \%$ nodes die in the network with initial energy $E$ 0 $=1 \mathrm{~J}$.

TABLE 7.1

Network lifetime of simulated protocols together with stability and instability periods

\begin{tabular}{|l|l|l|l|l|l|}
\hline Protocol & FND & HND & LND & $\begin{array}{l}\text { Stability } \\
\text { Period }\end{array}$ & $\begin{array}{l}\text { Instability } \\
\text { Period }\end{array}$ \\
\hline LEACH & 1805.2 & 2215.2 & 2763.5 & 1805.2 & 958.3 \\
\hline DRESEP & 4101.6 & 5126.7 & 6402.2 & 4101.6 & 2300.6 \\
\hline SEECP & 5109.5 & 5143.6 & 5154.3 & 5109.5 & $\mathbf{4 4 . 8}$ \\
\hline ZEERP & 4923.5 & $\mathbf{5 8 5 1 . 4}$ & $\mathbf{6 5 7 6 . 8}$ & 4923.5 & 1653.3 \\
\hline SZEERP & $\mathbf{5 4 9 3 . 3}$ & 5546.8 & 5573.2 & $\mathbf{5 4 9 3 . 3}$ & 79.9 \\
\hline
\end{tabular}

while minimizing the instability period. Usually, there is a tradeoff between stability and lifetime of the system. An optimal balance between these two contradictory goals is a challenge, and is essential to improve the overall network performance. To extend stability period and to balance the load effectively, another protocol named stable version of ZEERP (SZEERP) is proposed. The operation of the protocol is studied in terms of rounds, similar to ZEERP. The essential functioning of SZEERP is same as that of ZEERP. However, SZEERP uses residual energy as a parameter for ZH election (similar to SEECP) in selection and reselection phase for approaching robust performance. The deployment phase and zone formation phase follow the same procedure given in ZEERP. In SZEERP, ZH is selected on the basis of residual energy $\left(R_{E}\right)$ but not on average distance value $\left(A_{D}\right)$ as given in (6) for ZEERP. Similarly, in reselection phase for a given iteration, if $R_{E}$ of $\mathrm{ZH}$ is less than or equal to average energy of the respective zone, the corresponding ZH will be replaced by highest energy node of that zone.

7. Performance analysis of SZEERP. Figure 7.1 shows SZEERP outperforms ZEERP by increasing the stability period for a considerably higher number of rounds. By restricting $\mathrm{ZH}$ election criteria to choose the highest energy node, the energy level of all nodes are uniformly preserved throughout the simulation. SZEERP minimizes the energy variance of the network and provides an indistinguishable flat variance plot as shown in Figure 7.2.

Table 7.1 presents the number of rounds taken for FND, HND and LND for simulated protocols together with stability and instability periods. Figure 7.3 shows that there is an improvement of $204.32 \%, 33.94 \%, 9.87 \%$ 


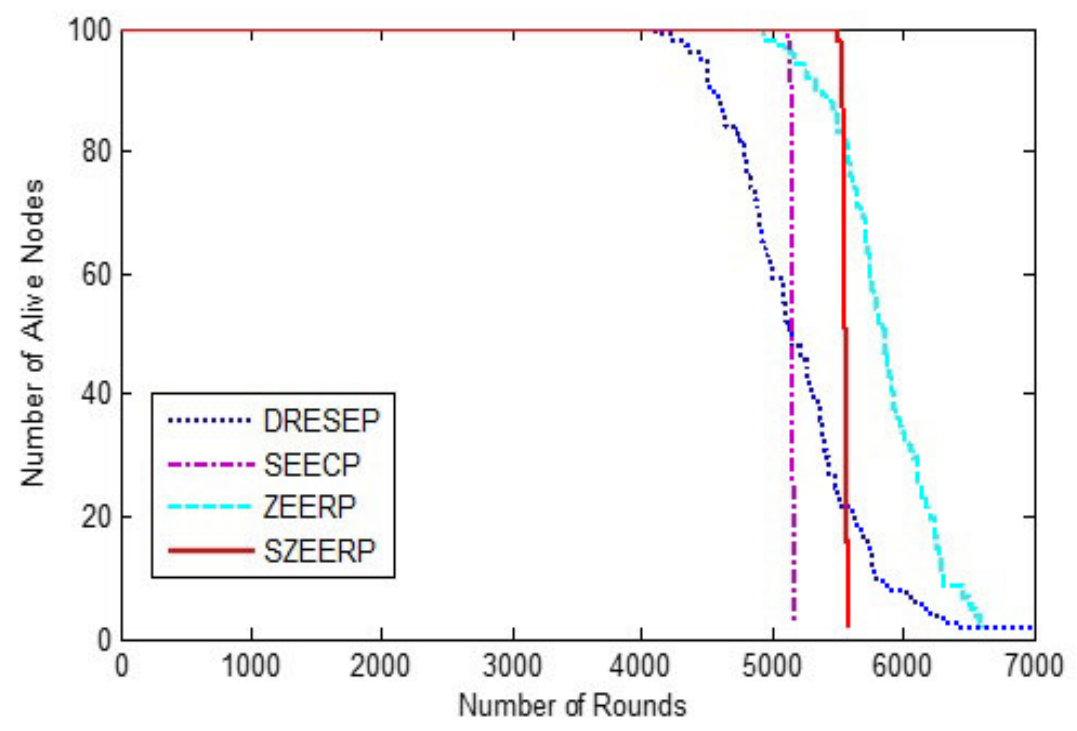

FiG. 7.1. Number of alive nodes per round for simulated protocols.

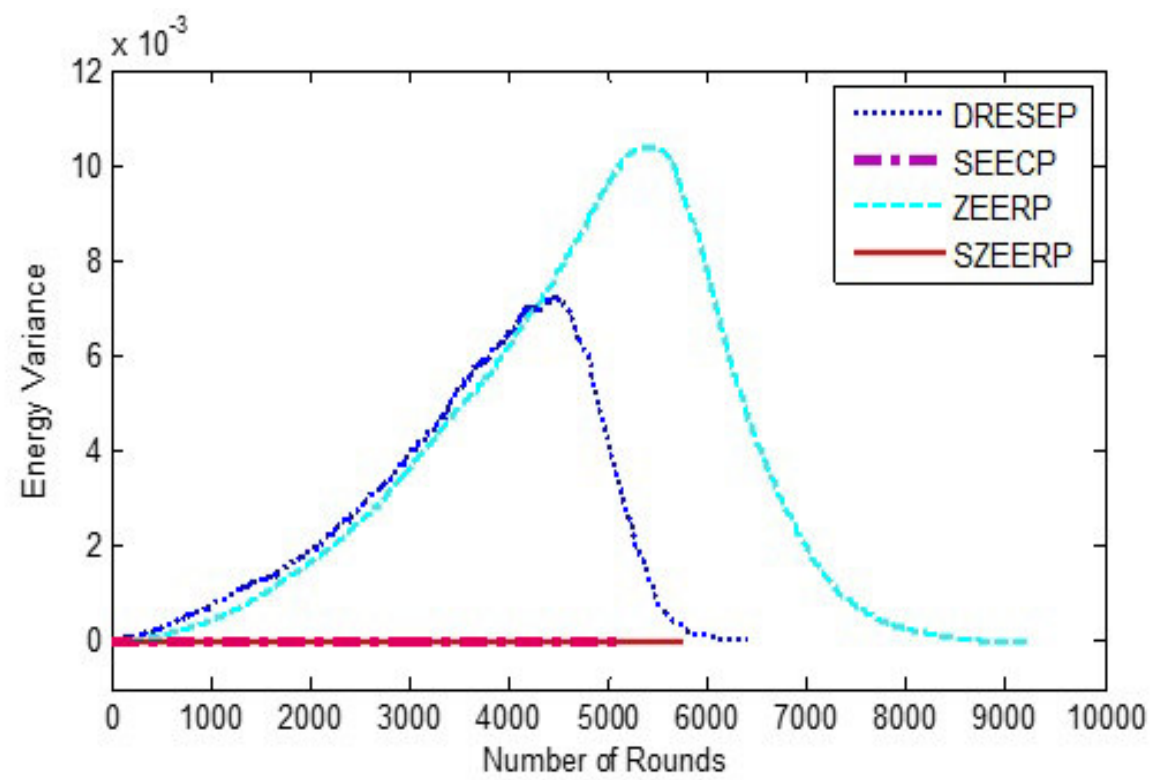

FIG. 7.2. Energy variance per round for simulated protocols.

and $11.57 \%$ in stability period for SZEERP as against LEACH, DRESEP, SEECP and ZEERP respectively. In addition, the instability period reduces to $96 \%, 98.33 \%$ and $98.77 \%$ in comparison with LEACH, DRESEP and ZEERP respectively. The results show that SZEERP is crucial for applications like military applications and health care applications in which each node is equally important and that require complete coverage of the network.

8. Conclusions and future scope. In this paper, a grid based randomly deployed sensor node network frame work is proposed that is well suited for time critical applications. The proposed approach evenly 


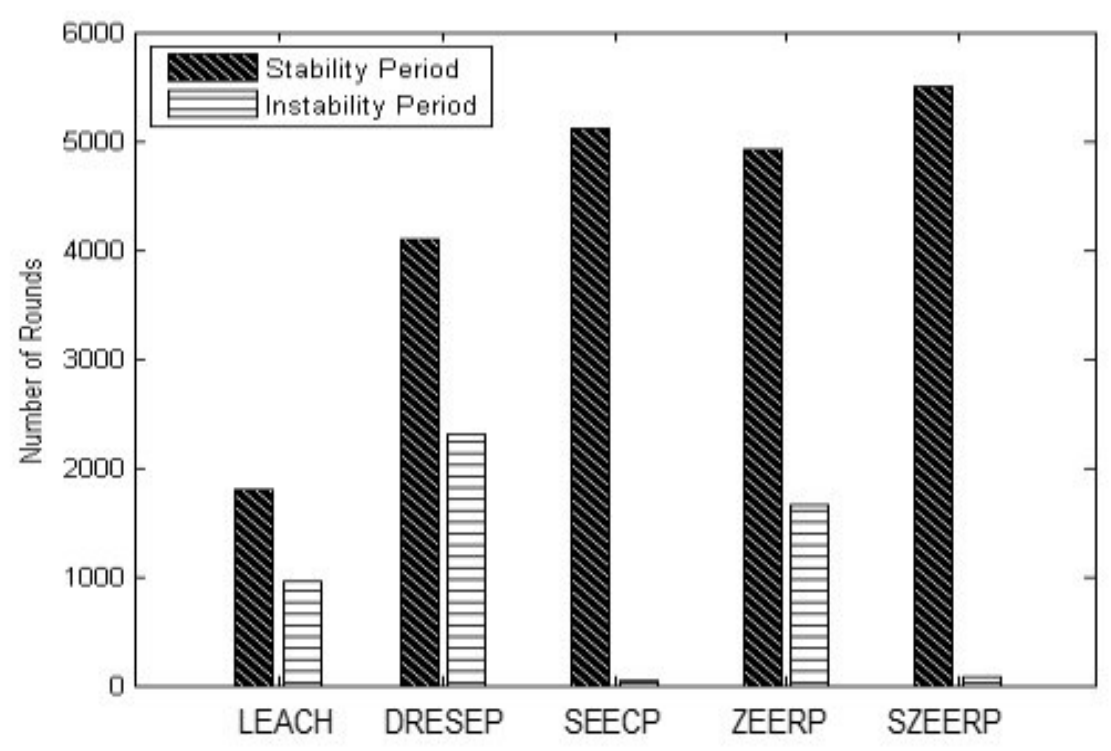

FIG. 7.3. Performance results of simulated protocols for network lifetime.

distributes the load across the network, improves network management, and extends network lifetime. The simulation results reveal that in ZEERP, total energy consumption during zone formation, ZH selection, reselection of ZH, and transmission has been reduced. In comparison with other existing protocols such as LEACH, TEEN and DRESEP, the proposed ZEERP protocol maximizes the lifetime approximately by $225.14 \%, 12.12 \%$ and $2.12 \%$ respectively. To extend stability period and to balance the load effectively, another protocolnamed stable version of ZEERP (SZEERP) is proposed. There is an improvement of $204.32 \%, 33.94 \%, 9.87 \%$ and $11.57 \%$ in the stability period for SZEERP in opposition to LEACH, DRESEP, SEECP and ZEERP respectively. In addition, the instability period for SZEERPs reduces to $96 \%, 98.33 \%$ and $98.77 \%$ in contrast to LEACH, DRESEP and ZEERP respectively. The proposed algorithms also outperform other approaches for parameters such as network lifetime, and total energy consumption.

The proposed algorithm opens a lot of gates for the future researchers. This work can be extended as an underlying topology for other energy efficient routing and load balancing protocols. In addition, further research is required to make the network framework adaptive by automatically optimizing number of grids, lower and upper bound for a specified number of senor nodes and terrain area. The future researchers may try their hands in improvising the selection and reselection process of Zone Head $(\mathrm{ZH})$ in order to increase efficiency and to prolong lifetime of sensor network. It would be also interesting to see the impact of evolutionary algorithms in the proposed technique.

\section{REFERENCES}

[1] Kıм, H. Y., An energy-efficient load balancing scheme to extend lifetime in wireless sensor networks. Cluster Computing, 19(1), 279283, 2016.

[2] Singh, A. M., Bhatia, T., Sharma, G., Shrivastava, G. "Artificial Intelligence Based Intrusion Detection System to Detect Flooding Attack in VANETs." In Handbook of Research on Network Forensics and Analysis Techniques, pp. 87-100. IGI Global, 2018.

[3] Shrivastava, G. Kumar, P., Gupta, B. B., Bala, S., Dey, N. Handbook of Research on Network Forensics and Analysis Techniques, 2018.

[4] Gupta, B. B., Joshi, R. C., Misra, M., Meena, D. L., Shrivastava, G., Sharma, K. Detecting a wide range of flooding DDoS attacks using linear prediction model, In IEEE 2nd International Conference on Information and Multimedia Technology (ICIMT 2010), vol. 2, pp. 535-539.2010.

[5] Arzoo, M., Bhatia, T., Sharma, G., Shrivastava, G. An Energy Efficient and Trust Aware Framework for Secure Routing 
in LEACH for Wireless Sensor Networks. Scalable Computing: Practice and Experience 18, no. 3 (2017): $207-218$.

[6] Chiti, F., Fantacci, R., Mastandrea, R., Rigazzi, G., Sarmiento, lvaro Surez, \& Lpez, E. M. M. . A distributed clustering scheme with self nomination: proposal and application to critical monitoring. Wireless Networks, 21(1), 329345, 2015 https://doi.org/10.1007/s11276-014- 0785-z

[7] Younis, M., Senturk, I. F., Akkaya, K., Lee, S., \& Senel, F. Topology management techniques for tolerating node failures in wireless sensor networks: A survey. Computer Networks (Vol. 58). Elsevier B.V.. 2014 https://doi.org/10.1016/j.comnet.2013.08.021

[8] LiU, X., A survey on clustering routing protocols in wireless sensor networks. Sensors (Switzerland) (Vol. 12), 2012 https://doi.org/10.3390/s120811113

[9] Zaman, N., Low, T. J., \& Alghamd, T, Enhancing routing energy efficiency of Wireless Sensor Networks, 17th International Conference on Advanced Communication Technology (ICACT), 4(2), 587595, 2015 https://doi.org/10.1109/ICACT.2015.7224928

[10] Chang, J.-Y., \& Ju, P.-H., An energy-saving routing architecture with a uniform clustering algorithm for wireless body sensor networks, Future Generation Computer Systems, 35, 128140, 2014 https://doi.org/10.1016/j.future.2013.09.012

[11] Heinzelman, W. R., Chandrakasan, A., \& Balakrishnan, H., Energy-efficient communication protocol for wireless microsensor networks. Proceedings of the 33rd Annual Hawaii International Conference on System Sciences, vol.1(c), 10, 2000, https://doi.org/10.1109/HICSS.2000.926982

[12] Manjeshwar, A., \& Agrawal, D. P. TEEN: A Routing Protocol for Enhanced Efficiency in Wireless Sensor Networks, Proceedings 15th International Parallel and Distributed Processing Symposium, 0(C), 2001 https://doi.org/10.1109/IPDPS.2001.925197

[13] Sharma, T. P., Joshi, R. C., \& Misra, M., GBDD: Grid Based Data Dissemination in Wireless Sensor Networks, 16th International Conference on Advanced Computing and Communications, 234240, 2008, https://doi.org/10.1109/ADCOM.2008.4760454

[14] Heinzelman, W. B., Chandrakasan, A. P., \& Balakrishnan, H. An application-specific protocol architecture for wireless microsensor networks. IEEE Transactions on Wireless Communications, 1(4), 660670, 2009, https://doi.org/10.1109/TWC.2002.804190

[15] Smaragdakis, G., Matta, I., \& Bestavros, A., SEP: A stable election protocol for clustered heterogeneous wireless sensor networks. Second International Workshop on Sensor and Actor Network Protocols and Applications (SANPA 2004), 111, 2004, https://doi.org/10.3923/jmcomm.2010.38.42

[16] Kumar, D., Aseri, T. C., \& Patel, R. B., EEHC: Energy efficient heterogeneous clustered scheme for wireless sensor networks, Computer Communications, 32(4), 662667, 2009 https://doi.org/10.1016/ j.comcom.2008.11.025

[17] Kumar, D., Aseri, T. C., \& Patel, R. B., Multi-hop communication routing (MCR) protocol for heterogeneous wireless sensor networks. International Journal of Information Technology, Communications and Convergence, 1(2), 130145, 2011, https://doi.org/10.1504/IJITCC.2011.039281

[18] Mittal, N., \& Singh, U., Distance-Based Residual Energy-Efficient Stable Election Protocol for WSNs. Arabian Journal for Science and Engineering, 40(6), 16371646, 2015, https://doi.org/10.1007/s13369-015-1641-x

[19] Mittal, N., Singh, U., \& Sohi, B. S., A stable energy efficient clustering protocol for wireless sensor networks, Wireless Networks, 18091821, 2017, https://doi.org/10.1007/s11276- 016-1255-6

[20] Attea, B. A., \& Khalil, E. A., A new evolutionary based routing protocol for clustered heterogeneous wireless sensor networks. Applied Soft Computing, 12(7), 19501957, 2012 https://doi.org/10.1016/ j.asoc.2011.04.007

[21] Mittal, N., Singh, U., Salgotra, R., \& Sohi, B. S., A boolean spider monkey optimization based energy efficient clustering approach for WSNs. Wireless Networks, 117, 2017, https://doi.org/10.1007/s11276-017-1459-4

[22] Mittal, N., Singh, U., \& Sohi, B. S., A Novel Energy Efficient Stable Clustering Approach for Wireless Sensor Networks, Wireless Personal Communications, 95(3), 29472971, 2017, https://doi.org/10.1007/ s11277-017-3973-1

[23] Mittal, N., Singh, U., Sohi, B. S., An Energy Aware Cluster-based Stable Protocol For Wireless Sensor Networks" Neural Computing and Applications (NCAA), pp 1-18, 2018.

[24] ZENG, J., A Clustering method of combining grid and genetic algorithm in wirless sensor netwroks, 216, 773779, 2013, https://doi.org/10.1007/978-1-4471-4856-2

[25] Pantazis, N. A., Nikolidakis, S. A., Vergados, D. D., \& Member, S., Energy-Ef fi cient Routing Protocols in Wireless Sensor Networks: A Survey, 15(2), 551591, 2013.

[26] Chen, C., He, Z., Sun, H., Kuang, J., BAi, D., \& Yang, C., A grid-based energy efficient routing protocol in Wireless Sensor Networks. International Symposium on Wireless and Pervasive Computing (ISWPC), 16, 2013, https://doi.org/10.1109/ISWPC.2013.6707444

[27] Ismail, W. Z. W., \& Manaf, S. A., Study on Coverage in Wireless Sensor Network using Grid Based Strategy and Particle Swarm Optimization, (December), 69, 2010.

[28] Sharma, R., Sohi, B. S., \& Mittal, N., Hierarchical Energy Efficient MAC protocol for Wireless Sensor Networks, International Journal of Applied Engineering Research, 12(24), pp 14727-14738, 2017.

[29] Sharma, R., Sohi, B. S., Kumar, Shakti., Ann based framework for energy efficient routing in multi-hop wsns, International Journal of Advanced Research in Computer Science, 8(5), 1298-1308, 2017.

[30] Sharma, R., \& Sohi, B. S., A Compartitve Study on MAC Protocols for Wireless Sensor Networks on Energy Reduction. Internatioanl Journal of Computer Science and Information Security, 15(11), 3540, 2017

[31] Rajan Sharma, B. S. S., The Impact of Dynamic Scaling on Energy Consumption at Node Level in Wireless Sensor Networks, $13(1), 175188,2018$.

[32] Ojha, R. P., Sanyal, G., Srivastava, P. K., \& Sharma, K., Design and Analysis of Modified SiQRS Model for Performance Study of Wireless Sensor Network. Scalable Computing: Practice and Experience, 18(3), 229241 
https://doi.org/10.12694/scpe.v18i3.1303

[33] Amar Singh, Shakti Kumar, Sukhbir Singh Walia, FW-AODV : An Optimized AODV Routing Protocol for Wireless Mesh Networks, International Journal of Advanced Research in Computer Science, Volume, Volume 8, No. 3, March April 2017, pp. 1131-1135,2017.

[34] Shakti Kumar, S.S. Walia, Amar Singh, Parallel Big Bang-Big Crunch Algorithm, International Journal of Advanced Computing, ISSN:2051-0845, Vol.46, Issue. 3, September 2013, pp. 1330-1335, 2013

[35] Shakti Kumar, Amar Singh, Sukhbir Singh Walia, Parallel Big Bang - Big Crunch Global Optimization Algorithm: Performance and its Applications to routing in WMNs, Wireless Personal Communications, Springer, SCI Indexed Journa,l, pp. 1601- 1618, 2018.

[36] Sharma, K., Bala, S., Bansal, H., \& Shrivastava, G., Introduction to the special issue on secure solutions for network in scalable computing. Scalable Computing, 18(3), 2017, https://doi.org/10.12694/scpe.v18i3.1299

[37] Amar Singh,Shakti Kumar, Sukhbir Singh Walia, P3PGA: Multi-Population 3 Parent Genetic Algorithm and its Application to Routing in WMNs, International Journal of Advanced Research in Computer Science, Volume, 8(5), pp. 968-975, 2017 .

[38] Mittal, N., Singh, U., \& Sohi, B. S, Harmony Search Algorithm Based Threshold-sensitive Energy-efficient Clustering Protocols For WSNs, Ad Hoc \& Sensor Wireless Networks, 36 (1-4), pp 149-174, 2017

Edited by: Khaleel Ahmad

Received: Aug 2, 2018

Accepted: Jan 18, 2019 